PAPER

\title{
Neurovascular relationship at the trigeminal root entry zone in persistent idiopathic facial pain: findings from MRI 3D visualisation
}

\author{
E Lang, R Naraghi, L Tanrikulu, P Hastreiter, R Fahlbusch, B Neundörfer, R Tröscher-Weber
}

See Editorial Commentary, p 1470

J Neurol Neurosurg Psychiatry 2005;76:1506-1509. doi: 10.1136/jnnp.2005.066084

See end of article for

authors' affiliations

Correspondence to:

Prof. Dr. E Lang,

Department of Neurology and Pain Center, University of Erlangen-Nuremberg, Schwabachanlage 6, D91054 Erlangen,

Germany; eberhard.lang@

rzmail.uni-erlangen.de

Received 21 February 2005

Revised version received 30 April 2005

Accepted 23 May 2005

\begin{abstract}
Background: Patients with atypical neuralgia or atypical facial pain have been surgically treated with microvascular decompression (MVD) of the trigeminal root entry zone (TREZ). There are no data regarding the sensitivity and specificity of a vessel-TREZ relationship as a cause of pain in patients with persistent idiopathic facial pain (PIFP) according to the definition given by the International Headache Society (IHS). Methods: The TREZ was visualised by 3D CISS MRI in 12 patients with unilateral PIFP according to the IHS criteria.

Results: The frequency of artery-TREZ, vein-TREZ, or vessel (artery/vein)-TREZ contacts on the symptomatic and asymptomatic sides did not differ significantly. On the symptomatic side, vessel-TREZ contact was found in $58 \%$ of patients (sensitivity). On the asymptomatic side, vessel-TREZ contact was absent in $33 \%$ of patients (specificity).

Conclusions: On the basis of the low sensitivity and specificity found in the present study, PIFP cannot be attributed to a vessel-TREZ contact, and therefore, pain relief after MVD cannot be expected.
\end{abstract}

$\mathrm{P}$ ersistent idiopathic facial pain (PIFP), the term that has replaced the former "atypical facial pain", ${ }^{1}$ is defined as persistent facial pain that does not have the classic characteristics of cranial neuralgias and is not attributable to another disorder. Usually, PIFP starts in one quadrant of the face and spreads over time to neighbouring regions on the same side of the face or across the midline to the opposite side. ${ }^{2}$ Pain is deep and poorly localised, typically reported as continuous with constant or fluctuating intensity and described as burning, aching or cramping. The pain cannot be triggered or aggravated by remote stimuli. Clinical neurological examination of the trigeminal system usually does not initially reveal a sensory deficit.

The pathophysiology of PIFP is largely unknown. Psychiatric, ${ }^{3}$ vascular, ${ }^{4}$ central $^{5-7}$ and peripheral ${ }^{8}$ neurophysiological mechanisms have all been suggested. Furthermore, it has been hypothesised that trigeminal pain syndromes represent a continuum from typical to atypical neuralgia rather than discrete diagnoses. ${ }^{9}$ Based on a similar concept, microvascular decompression (MVD) of the trigeminal root entry zone (TREZ) from an offending vessel was performed in 672 patients with atypical trigeminal neuralgia and complete pain relief was reported in $51 \%$ of patients for up to five years after the surgery..$^{10}$ There are several other reports on small groups of patients suggesting that vascular compression may play a role in atypical facial pain, and that MVD may offer relief from this intractable condition. ${ }^{11-13}$ Vascular compression was reported in two of three (66\%) patients by Panagopoulos et al $^{12}$ and in 13 of 26 patients $(50 \%)$ by Antic et al. ${ }^{11}$ Unfortunately, these studies did not report whether the patients had atypical trigeminal neuralgia or trigeminal neuropathy according to the Liverpool criteria ${ }^{14}$ and whether the patients fulfilled the International Headache Society (IHS) criteria for PIFP. Nevertheless, data about the importance of a vessel-nerve conflict at the TREZ in patients fulfilling IHS criteria for PIFP would be useful for enhancing our knowledge of the pathophysiology of PIFP and, consequently, counselling patients regarding therapy.

To this end, we investigated the frequency of vessel-TREZ contact on the symptomatic and asymptomatic sides in patients with unilateral PIFP by means of a three dimensional magnetic resonance imaging visualisation (MRI 3D visualisation) technique. ${ }^{15}$

\section{METHODS}

\section{Patients}

We recruited 12 consecutive patients with unilateral PIFP ( six women, six men; mean (SD) age 48 (12) years) from the outpatient facility for pain management at the Department of Neurology, University of Erlangen-Nuremberg, Germany. The IHS diagnostic criteria (code 13.18.4) were used for inclusion of patients :

(A) Pain in the face, present daily and persisting for all or most of the day, fulfilling criteria $\mathrm{B}$ and $\mathrm{C}$

(B) Pain is confined at onset to a limited area on one side of the face, and is deep and poorly localised

(C) Pain is not associated with sensory loss or other physical signs

(D) Investigations including $x$ rays of the face and jaws do not demonstrate any relevant abnormality

In all patients, a specific cause of the pain was excluded by a thorough clinical examination by a neurologist, an otorhinolaryngologist, and an orodental specialist. All patients had normal findings on computed tomography (CT) or MRI scan of the head. The study followed the tenets of the Declaration of Helsinki. Ethics committee approval was

Abbreviations: CISS, constructive interference in steady state; IHS, International Headache Society; MVD, microvascular decompression; PIFP, persistent idiopathic facial pain; TREZ, trigeminal root entry zone 
not necessary because the MRI studies were undertaken for clinical diagnostic reasons, and because MRI is completely non-invasive. All participants gave their informed consent prior to the measurements.

\section{D visualisation of TREZ}

We performed 3D visualisation as has been previously described in detail..$^{15}$ Briefly, the neurovascular relationship at the TREZ was imaged in a head coil on a Siemens Sonata 1.5 T MR unit (Medical Solutions, Erlangen, Germany) using MR constructive interference in steady state (CISS) 3D and arterial magnetic resonance angiography time of flight 3D (MRA TOF) sequences of $0.4 \mathrm{~mm}$ slice thickness resulting in an iso-voxel size of $0.4 \mathrm{~mm}$. Explicit segmentation of the relevant structures in each slice included noise reduction by anisotropic diffusion, extraction of the cerebrospinal fluid space by volume growing, manual labelling of the cranial nerves and vessels, and attribution of different colours to the segmented subvolumes. Finally, the subvolumes of each slice were reconstructed by means of implicit segmentation to a $3 \mathrm{D}$ object visualising the brain stem, the entry zones of the cranial nerves $\mathrm{V}-\mathrm{X}$, and the vessels.

\section{Evaluation of the nerve-vessel contact}

A radiologist and an experienced research assistant independently analysed subsequent 2D MR CISS images and 3D visualisations, respectively. Both persons were blinded as regards the symptomatic side of PIFP. We defined TREZ as the segment of the trigeminal nerve, $6 \mathrm{~mm}$ in length, situated adjacent to the pons, which corresponds to the longest reported length of the central myelin. ${ }^{16}$ The extent of the vessel-TREZ relationship was categorised as: absence of contact (0), presence of contact (1), contact with deviation of the TREZ (2), and compression of the TREZ (3). Arteries were identified in the $3 \mathrm{D}$ visualisation as long curved vessels which could be traced back to their parent vessels. In contrast, veins in contact with the TREZ were visualised as extraneural hypointense signals that could not be traced over long distances and were mostly found posterolateral to the TREZ. Comparison of preoperative 3D visualisation with the corresponding intraoperative photomicrographs revealed good concordance. ${ }^{15}$

\section{Statistical evaluation}

Vessel-TREZ contact was categorised as "true positive" if a contact between an artery or a vein and the TREZ was observed on the symptomatic side. Vessel-TREZ contact was categorised as "true negative" if no contact was observed between an artery or a vein and the TREZ on the asymptomatic side. Sensitivity of the vessel-TREZ contact was calculated as the percentage of the true positive vesselTREZ contacts divided by the sum of true positive and false negative vessel-TREZ contacts (symptomatic side). Specificity of the vessel-TREZ contact was calculated as the percentage of the true negative vessel-TREZ contacts divided by the sum of the true negative and false positive vesselTREZ contacts (asymptomatic side). The frequencies of vessel-TREZ contact on the symptomatic and asymptomatic sides were compared with McNemar's test, and $\mathrm{p}<0.05$ was considered as statistically significant.

\section{RESULTS}

\section{Patients}

Pain was localised to the right side of the face in five patients and on the left side in seven patients. All patients felt the pain within the depth of the maxilla. Four patients also felt pain superficially in the skin. The pain was continuously present in all patients. The severity of pain in the preceding three months was rated on a numeric rating scale $(0=$ no pain, $10=$ worst pain) with an average (SD) of 6.2 (1.7). Its character was described as aching $(n=5)$, burning $(n=3)$, gnawing $(n=3)$, throbbing $(n=2)$, pressing $(n=2)$, stinging, $(\mathrm{n}=2)$ or dull $(\mathrm{n}=1)$. The pain was not triggered by remote stimuli. The average duration of pain was 106 (94) months (range 10-300 months). At the time of onset of the pain three patients reported having dental treatment or oromaxillary surgery, one patient a heavy blow to the left zygomatic arch, whereas eight patients could not remember any event. Eleven patients had undergone extraction of teeth for relief of pain but this was unsuccessful (median number of teeth extracted $=2$ ). In addition four patients underwent two to six surgical interventions in the oromaxillary region and this too did not relieve the pain. Neurological evaluation of the face did not reveal any sensory deficits.

\section{Neurovascular relationship at the TREZ}

In $11 / 12$ patients, the finding of an artery-TREZ contact by the two independent examiners coincided exactly. In the twelfth patient, artery-TREZ contact was visible only after segmentation and 3D visualisation. In the present study, vein-TREZ contacts have been described in 3D reconstructions only. A typical specimen of a 3D visualisation is shown in fig 1. Vessel-TREZ contacts on the symptomatic and asymptomatic sides for each patient are listed in table 1 and the frequencies are summarised in table 2 . The frequency of vessel-TREZ contact did not differ $(p>0.9)$ between the symptomatic and asymptomatic sides. None of the patients had any deviation or compression at the TREZ.

Sensitivity of the vessel-TREZ contact (symptomatic side) was $58 \%$, and the specificity of vessel-TREZ contact (asymptomatic side) was 33\%. Corresponding data for the artery-TREZ contacts and vein-TREZ contacts are given in table 2 .

One patient with an artery-TREZ contact on the symptomatic side only wished to undergo MVD (multiple cranial nerve decompression including trigeminal nerve and nervus intermedius in another hospital). However, this patient's pain did not improve after surgery.

\section{DISCUSSION}

Compared to intraoperative microphotography during MVD for trigeminal neuralgia, preoperative MRI 3D visualisation is known to be a sensitive method for visualising a possible vessel-TREZ contact. ${ }^{15} 17$ We found that 3D visualisation

Table 1 Absence of contact (0) or contact (1) of the artery-trigeminal root entry zone (TREZ) or vein-TREZ relationship on the symptomatic and asymptomatic sides in patients with unilateral persistent idiopathic facial pain (3D magnetic resonance imaging visualisation)

\begin{tabular}{|c|c|c|c|c|}
\hline \multirow[b]{2}{*}{$\begin{array}{l}\text { Patient } \\
\text { no }\end{array}$} & \multicolumn{2}{|c|}{ Artery-TREZ contact } & \multicolumn{2}{|c|}{ Vein-TREZ contact } \\
\hline & $\begin{array}{l}\text { Symptomatic } \\
\text { side }\end{array}$ & $\begin{array}{l}\text { Asymptomatic } \\
\text { side }\end{array}$ & $\begin{array}{l}\text { Symptomatic } \\
\text { side }\end{array}$ & $\begin{array}{l}\text { Asymptomatic } \\
\text { side }\end{array}$ \\
\hline 1 & 0 & 0 & 0 & 1 \\
\hline 2 & 1 & 1 & 0 & 1 \\
\hline 3 & 0 & 0 & 1 & 1 \\
\hline 4 & 1 & 1 & 1 & 0 \\
\hline 5 & 0 & 0 & 0 & 0 \\
\hline 6 & 1 & 0 & 0 & 1 \\
\hline 7 & 0 & 0 & 0 & 0 \\
\hline 8 & 0 & 1 & 1 & 0 \\
\hline 9 & 0 & 0 & 0 & 1 \\
\hline 10 & 0 & 0 & 0 & 0 \\
\hline 11 & 0 & 1 & 1 & 1 \\
\hline 12 & 1 & 0 & 0 & 0 \\
\hline Total & 4 & 4 & 4 & 6 \\
\hline
\end{tabular}



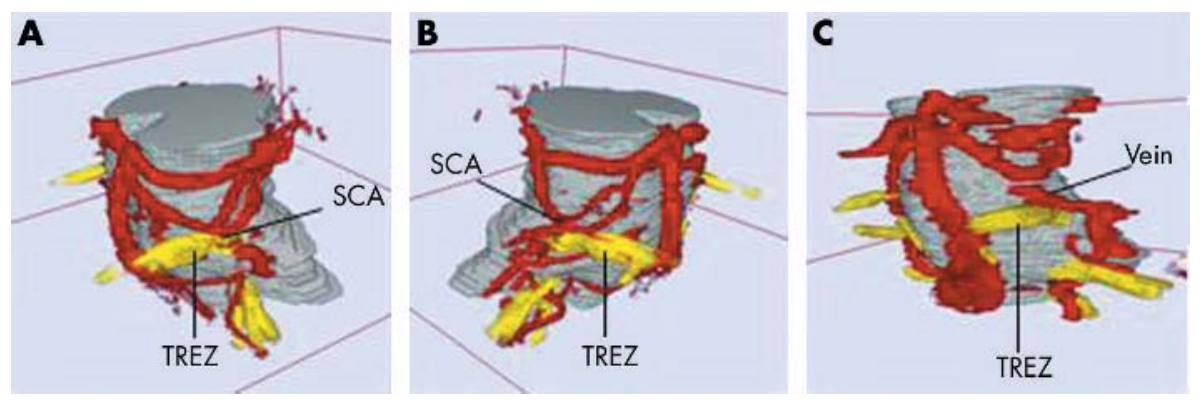

Figure 1 Magnetic resonance imaging (MRI) three dimensional visualisation of the superior cerebellar artery (SCA)-trigeminal root entry zone (TREZ) contact on the (A) symptomatic and $(B)$ asymptomatic sides in patient no 4. (C) Vein-TREZ contact on the asymptomatic side in patient no 6 . following segmentation was superior to evaluation of 2D MRI CISS images because the former identified an artery-TREZ contact missed by the 2D MRI CISS. In addition, 3D visualisation also allowed vein-TREZ contacts to be identified. However, in patients with PIFP diagnosed according to the IHS criteria, ${ }^{1}$ 3D visualisation of the TREZ revealed true positive findings on the symptomatic side in only $58 \%$ (sensitivity) and true negative findings on the asymptomatic side in only 33\% (specificity). In addition, the frequency of vessel-TREZ contact did not differ between the symptomatic and asymptomatic sides. Because of the low discriminative function of the presence of a vessel-TREZ contact, it seems unlikely that vessel-TREZ contact and pain are causally related in patients with PIFP.

We chose the presence of any simple vessel-TREZ contact on the symptomatic side as the true positive condition because only those patients who have trigeminal neuralgia with neurovascular contact have been reported to benefit from MVD. ${ }^{18}$ Grooving, distortion, or deviation of the trigeminal root, which has been reported to be more specific for idiopathic trigeminal neuralgia, ${ }^{19}$ was not observed in the present patient group. This is in agreement with a study which reported rare vessel-TREZ compression in patients with atypical facial pain. ${ }^{11}$

We included vein-TREZ contacts in the analysis since firstly, the success rate of vein decompression in trigeminal neuralgia is almost as high as that of pure arterial compression with regard to pain relief, ${ }^{20}$ and secondly, venous compression of the TREZ has been reported in patients with atypical neuralgia. ${ }^{21}$ Unfortunately, a weakness of the MRI technique that we used is that MR CISS cannot differentiate between arteries and veins or veins and other tissues that produce a hypointense signal. However, taking into consideration anatomical knowledge of the important vein complex that lies lateral to the trigeminal nerve and the characteristic appearance of an extraneural hypointense signal from the surrounding cerebrospinal fluid, intraoperative photomicrographs in a previous study did not reveal unexpected findings. ${ }^{15}$

Table 2 Frequency of vessel-trigeminal root entry zone (TREZ) contact on the symptomatic and asymptomatic sides in patients. Sensitivity (symptomatic side) and specificity (asymptomatic side) in the subgroups of vesselTREZ contacts are given in parentheses

\begin{tabular}{|c|c|c|c|c|}
\hline Relationship & Contact & $\begin{array}{l}\text { Symptomatic } \\
\text { side }\end{array}$ & $\begin{array}{l}\text { Asymptomatic } \\
\text { side }\end{array}$ & $\begin{array}{l}\text { p(McNemar } \\
\text { test) }\end{array}$ \\
\hline \multirow[t]{2}{*}{ Artery-TREZ } & Present (1) & $4(33 \%)$ & 4 & \\
\hline & Absent (0) & 8 & $8(66 \%)$ & 1.0 \\
\hline \multirow[t]{2}{*}{ Vein-TREZ } & Present (1) & $4(33 \%)$ & 6 & \\
\hline & Absent (0) & 8 & $6(50 \%)$ & $>0.3$ \\
\hline \multirow[t]{2}{*}{ Artery/vein-TREZ } & Present (1) & $7(58 \%)$ & 8 & \\
\hline & Absent (0) & 5 & $4(33 \%)$ & $>0.9$ \\
\hline
\end{tabular}

The frequency of vessel-TREZ contacts in PIFP on the symptomatic side was found to lie within the range of coincidental findings observed in asymptomatic subjects or sides. In studies on cadavers of individuals who were known not have been affected by trigeminal neuralgia, vessel-nerve contact was reported in $58 \%,{ }^{22} 35 \%,{ }^{23} 32.4 \%,{ }^{24}$ and $39 \%$ of examined TREZs. A large MR study of 85 asymptomatic subjects (170 trigeminal nerves) revealed that in 30\% there was vessel-TREZ contact. ${ }^{26}$ This is an older study and it must be considered that, compared with the present study, the resolution of the MR technique was lower. In another MRI study on patients with trigeminal neuralgia, a simple vesselTREZ contact on the asymptomatic side was observed in 39\% of patients. ${ }^{19}$

Patients with PIFP differ from those with a previous history of trigeminal neuralgia that may have developed into an atypical trigeminal neuralgia or trigeminal neuropathy. ${ }^{14}$ However, in the large outcome study of Tyler-Kabara and colleagues ${ }^{10}$ patients were not differentiated by their history, actual pain characteristics, and sensory findings. Hence outcomes cannot be generalised to patients with PIFP. One of our patients in the present study who wished to undergo MVD did not benefit from multiple cranial nerve decompression including that of the trigeminal nerve and nervus intermedius. It remains open whether there is a link between the success rate of $51 \%$ in Jannetta's group ${ }^{10}$ and our positive findings of $58 \%$ on the affected side. Additional contributing factors must be considered.

As indicated by the frequent vessel-TREZ contacts in asymptomatic subjects or in patients with trigeminal neuralgia on the asymptomatic side of the face, vesselTREZ contact in trigeminal neuralgia may be a necessary but not sufficient condition. Given the absence of definitive criteria distinguishing "significant" from "incidental" compression, the finding of a vessel-TREZ contact in the preoperative MRI in patients with an appropriate diagnosis of trigeminal neuralgia on clinical grounds must be assumed to be significant and, therefore, relevant for planning therapy. ${ }^{27}$ It may be the characteristic clinical signs and symptoms occurring in the course of the idiopathic trigeminal neuralgia that indicate the presence of unknown cofactors of the symptomatic vessel-TREZ contact and therefore can predict patients' good response to MVD.

It should, therefore, be emphasised that MRI cannot be used as a convenient tool for either making a diagnosis or planning therapy for trigeminal neuralgia unless supported by a clinical diagnosis based on sound criteria. The risks of using MRI findings of vessel-TREZ contact for diagnosis apply in particular to other chronic orofacial pain states that are more common $\left(23 \%^{28}\right)$ than the relative rare trigeminal neuralgia $\left(0.0043 \%{ }^{29}\right)$, because a vessel-TREZ contact on MRI alone has no proved pathophysiological significance and should be regarded as incidental. To our knowledge, MVD for the treatment of PIFP as defined by the IHS criteria ${ }^{1}$ has never been shown to be effective in a controlled clinical study. 
We conclude that PIFP, as defined by the IHS criteria, ${ }^{1}$ is not related to vessel-TREZ contact. In the present study, neither compression nor deviation of the TREZ was found. Therefore, patients with PIFP cannot be expected to have pain relief after MVD of the vessel-TREZ contact.

\section{Authors' affiliations}

E Lang, B Neundörfer, Department of Neurology and Pain Center, University of Erlangen-Nuremberg, Erlangen, Germany

R Naraghi, L Tanrikulu, R Fahlbusch, Department of Neurosurgery and Neurocenter, University of Erlangen-Nuremberg, Erlangen, Germany

P Hastreiter, Neurocenter, University of Erlangen-Nuremberg, Erlangen, Germany

R Tröscher-Weber, Department of Neurosurgery and Division of Neuroradiology, University of Erlangen-Nuremberg, Erlangen,

Germany

Competing interests: none declared

\section{REFERENCES}

1 International Headache Society Classification Subcommittee. International classification of headache disorders, 2nd edn. Cephalalgia 2004;24(suppl 1):1-160.

2 Sharav Y. Orofacial pain. In: Wall PD, Melzack R, eds. Textbook of pain, 4th edn. Edinburgh: Churchill Livingstone, 1999:728.

3 Feinmann C, Harris M, Cawley R. Psychogenic facial pain: presentation and treatment. Br Med J 1984;288:436-8.

4 Harrison SD, Balawi SA, Feinmann C, et al. Atypical facial pain: a double blind placebo-controlled crossover pilot study of subcutaneous sumatriptan. Eur Neuropsychopharmacol 1997;7:83-8.

5 Derbyshire SWG, Whalley MG, Stenger VA, et al. Cerebral activation during hypnotically induced and imagined pain. Neuroimage 2004;23:392-401.

6 Jääskeläinen SK, Forsell H, Tenovuo O. Electrophysiological testing of the trigeminofacial system: aid in the diagnosis of atypical facial pain. Pain 1999;80:191-200.

7 Hagelberg $\mathrm{N}$, Forssell $\mathrm{H}$, Aalto $\mathrm{S}$, et al. Altered dopamine D2 receptor binding in atypical facial pain. Pain 2003;106:43-8.

8 Ziccardi VB, Janosky JE, Patterson GT, et al. Peripheral trigeminal nerve surgery for patients with atypical pain. J Craniomaxillofac Surg 1994;22:355-60.

9 Burchiel KJ, Slavin KV. On the natural history of trigeminal neuralgia. Neurosurgery 2000;46:152-5.

10 Tyler-Kabara EC, Kassam AB, Horowitz MH, et al. Predictors of outcome in surgically managed patients with typical and atypical trigeminal neuralgia: comparison of results following microvascular decompression. J Neurosurg 2002;96:527-31

11 Antic B, Markovic U, Savic M. Abnormality of the root entry zone as a cause of typical and atypical trigeminal neuralgia. J Neurol Sci 1997; 150(suppl 1):S201.

12 Panagopoulos K, Chakraborty M, Deopujari CE, et al. Neurovascular decompression for cranial rhizopathies. Br J Neurosurg 1987; 1:235-41.

13 Shi-Ting L, Quinggang P, Ningtao L, et al. Trigeminal neuralgia: What are the important factors for good operative outcomes with microvascular decompression. Surg Neurol 2004;62:400-5.

14 Nurmikko TJ, Eldridge PR. Trigeminal neuralgia-pathophysiology, diagnosis and current treatment. Br J Anaesth 2001;87:117-32.

15 Naraghi R, Hastreiter P, Tomandl B, et al. Three-dimensional visualization of neurovascular relationships in the posterior fossa: technique and clinical application. J Neurosurg 2004;100:1025-35.

16 Lang J. Clinical anatomy of the posterior cranial fossa and its foramina. Stuttgart: Georg Thieme Verlag, 1991:82.

17 Yoshino N, Akimoto H, Yamada I, et al. Trigeminal neuralgia: Evaluation of neuralgic manifestation and site of neurovascular compression with 3D CISS MR imaging and MR angiography. Radiology 2003;228:539-45.

18 Burchiel KJ, Clarke $\mathrm{H}$, Haglund $M$, et al. Long-term efficacy of microvascular decompression in trigeminal neuralgia. J Neurosurg 1988;69:35-8

19 Masur H, Papke K, Bongartz G, et al. The significance of three-dimensional MR-defined neurovascular compression for the pathogenesis of trigeminal neuralgia. J Neurol 1995;242:93-8.

20 Barker FG II, Jannetta PJ, Bissonette DJ, et al. The long-term outcome of microvascular decompression for trigeminal neuralgia. N Engl J Med 1996;334:1077-83.

21 Lee SH, Levy El, Scarrow AM, et al. Recurrent trigeminal neuralgia attributable to veins after microvascular decompression. Neurosurgery 2000;46:356-61.

22 Hardy DG, Rhoton AL. Microsurgical relationship of the superior cerebellar artery and the trigeminal nerve. J Neurosurg 1978;49:669-78.

23 Haines SJ, Jannetta PJ, Zorub DS. Microvascular relations of the trigeminal nerve. J Neurosurg 1980;52:381-6.

24 Klun B, Prestor B. Microvascular relations of the trigeminal nerve: an anatomical study. Neurosurgery 1986;19:535-8.

25 Hamlyn PJ. Neurovascular compression of the lower cranial nerves. Amsterdam: Elsevier, 1999:117.

26 Tash RR, Sze G, Leslie DR. Trigeminal neuralgia: MR imaging features. Radiology 1989:172:767-70.

27 Meaney JFM, Eldridge PR, Dunn LT, et al. Demonstration of neurovascular compression in trigeminal neuralgia with magnetic resonance imaging. J Neurosurg 1995;83:799-805.

28 Macfarlane TV, Blinkhorn AS, Davies RM, et al. Orofacial pain: just another chronic pain? Results from a population-based survey. Pain 2002;99:453-8.

29 Katusic S, Beard CM, Bergstralh E, et al. Incidence and clinical features of trigeminal neuralgia, Rochester, Minnesota, 1945-1984. Ann Neurol 1990;27:89-95. 